\title{
Kolorimetrischer Nachweis von Chondroitinsulfat B im Gemisch mit anderen sauren Mucopolysacchariden
}

\author{
Von W. TELLER $\left.{ }^{1}\right)$ \\ Aus der Universitäts-Kinderklinile Marburg a.d. Labn (Direktor: Prof. Dr. F. Linneweb)
}

(Eingegangen am 5. April 1966)

\begin{abstract}
Es wurde das Verhalten von drei Hexuronsäuren (D-Glukuronsäure, L-Iduronsäure, D-Galakturonsäure) und vier gereinigten sauren Mucopolysacchariden (Chondroitinsulfat A,B, C und Heparitinsulfat) in der Carbazol-und in der Naphthoresorcin-Reaktion untersucht. Dabei fiel die geringe Farbentwicklung von L-Iduronsäure und Chondroitinsulfat B in der Carbazol-Reaktion auf. In der Naphthoresorcin-Reaktion zeigten diese Substanzen eine ausgeprägte Chromogenität. In einem Gemisch aus Chondroitinsulfat A, C und Heparitinsulfat konnte bei konstanter Gesamtmenge die Farbentwicklung durch steigenden Zusatz von Chondroitinsulfat B in der Carbazol-Reaktion linear vermindert, in der Naphthoresorcin-Reaktion linear gesteigert werden. Für verschiedene prozentuale Mengen von Chondroitinsulfat B im Gemisch mehrerer saurer Mucopolysaccharide wurden die Carbazol/Naphthoresorcin(,,C/N")-Quotienten ermittelt. Sie standen zueinander in einfacher exponentieller Beziehung. Der mittlere $\mathrm{C} / \mathrm{N}-\mathrm{Quo}-$ tient der sauren Mucopolysaccharide im Harn von 37 Normalpersonen betrug 15,1 $\pm 5,6(M \pm \sigma)$. 13 Patienten mit typischer Pfaundler-Hurler'scher Krankheit wiesen einen mittleren $C / N-Q u o t i e n t e n$ von 4,2 $\pm 1,2(M \pm \sigma)$ auf. Dieser signifikant unterhalb der Norm liegende Wert ist durch eine vermehrte Ausscheidung von Chondroitinsulfat $B$ bei Patienten mit Gargoylismus bedingt.
\end{abstract}

The chromogenicities of three hexuronic acids (D-glucuronic acid, L-iduronic acid, D-galacturonic acid) and four acid mucopolysaccharides (chondroitin sulfate $A, B, C$ and heparitin sulfate) were examined in the carbazole as well as in the naphthoresorcinol reaction. L-iduronic acid and chondroitin sulfate $B$ revealed only slight color development in the carbazole reaction, while in the naphtho resorcinol reaction their color yield was considerable. The stepwise addition of chondroitin sulfate $B$ to a mixture containing constant amounts of chondroitin sulfates $A, C$ and heparitin sulfate caused a linear depression of color development in the carbazole reaction and a linear increase of optical density in the naphthoresorcinol reaction. Carbazole/naphthoresorcinol (,C/N") ratios were determined for several mixtures of acid mucopolysaccharides containing various percentages of chondroitin sulfate $B$. These $C / N$ ratios followed a linear function on a semilogarithmic scale. 37 normal persons revealed a urinary acid mucopolysaccharide excretion with the mean $\mathrm{C} / \mathrm{N}$ ratio of $15.1 \pm 5.6(\mathrm{M} \pm \sigma)$. In 13 patients with gargoylism the $\mathrm{C} / \mathrm{N}$ ratio of urinary acid mucopolysaccharides was $4.2 \pm 1.2(\mathrm{M} \pm \sigma)$. This decreased $\mathrm{C} / \mathrm{N}$ ratio appears to be due to the increased excretion of chondroitin sulfate B by patients with Hurler's syndrome.

In Zusammenhang mit der Feststellung einer Mucopolysaccharidurie bei der Pfaundler-Hurler'schen Krankheit (Gargoylismus) durch Dorfman und Lorincz (1), sowie MEYER und Mitarbeiter (2) hat sich bei Patienten mit enchondralen Dysostosen die Notwendigkeit einer quantitativen Mucopolysaccharid-Bestimmung im Harn ergeben. In zahlreichẹn Fällen von Gargoylismus scheint sie das zuverlässigste diagnostische Hilfsmittel zu sein (3). Die säulen- und/oder papierchromatographische Fraktionierung der sauren Mucopolysaccharide („sMP“c) im Harn hat weiterhin eine Einteilung der PfaundlerHurler'schen Krankheit in 5 verschiedene Gruppen mit einem jeweils charakteristischen Ausscheidungsmuster der SMP ermöglicht (4). Allerdings sind die verwandten Methoden aufweridig und nicht in jedem Laboratorium durchführbar.

Da die typische Form des Gargoylismus mit einer abnormen Ausscheidung von Chondroitinsulfat B (Dermatansulfat) und Heparitinsulfat (Heparansulfat) einhergeht $(1,2)$, ist es bei Patienten mit Verdacht auf enchondrale Dysostosen von klinisch-chemischer Bedeutung, neben der quantitativ erhöhten Mucopolysaccharidausscheidung auch eines dieser beiden qualitativ nachzuweisen. Die Methoden sollten dabei relativ einfach und in klinischen Routinelabotatorien anwendbar sein.

1) Mit dankenswerter Unterstïtzung der Deutschen Forschungsgemeinschaft, Bad Godesberg.
Der Nachweis von Chondroitinsulfat B ("CSB") in einem Gemisch mehrerer Mucopolysaccharide gelingt bei der Carbazol-Methode durch Veränderung der Reaktionsbedingungen (5). Auch durch die Verwendung zweier oder mehrerer kolorimetrischer Verfahren, in denen CSB gegenüber anderen SMP unterschiedliche Farbentwicklung (Chromogenität) besitzt, läßt sich dieses, normalerweise nur in Spuren im Harn vorkommende Mucopolysaccharid bestimmen. In der vorliegenden Arbeit soll über unsere Versuche zur gleichzeitigen Anwendung der Carbazol- und der Naphthoresorcin-Reaktion zum Nachweis von Chondroitinsulfat B in Mucopolysaccharid-Gemischen und im Harn berichtet werden.

\section{Methodik}

Reagenzien

Hexuronsäuren

D-Glukuronsäure, purum (Fa. Fluka); D-Galakturonsäure, puriss. (Fa. Flụka); L-Iduronsäure, wurde uns freundlicherweise von Herrn Professor Wolfrom, Ohio State University, Columbus, Ohio (USA) zur Verfügung gestellt.

Gereinigte Saure Mucopolysaccharide

Für ihre Uberlassung sind wir Herrn Professor K. Meyer, Columbia University, New York (USA) zu großem Dank verpflichtet. Chondroitinsulfat A (Chondroitin-4-Sulfat ${ }^{1}$ ) (CSA)

Chondroitinsulfat C (Chondroitin-6-sulfat ${ }^{1}$ ) (CSC)

Chondroitinsulfat B (Dermatansulfat ${ }^{1}$ ) (CSB). .

Heparitinsulfat (Heparansulfat ${ }^{1}$ ) (HMS).

1) Bezeichnung gemäß des Vorschlages von Herrn Professor MEYER, New York, zur Polysaccharid-Nomeñklatur, American Chemical Society, Mai 1959. 


\section{Sonstige}

Naphthoresorcin, puriss. (Fa. Fluka)

Carbazol, sublimiert, purum (Fa. Fluka)

Cetavlon (CTAB): $n$-Hexadecyltrimethylammoniumbromid, purum (Fa. Fluka)

Dialysierschlauch (Fa. Kalle und Co., Wiesbaden)

\section{Kolorimetrische Reaktionen}

Hexuronsäurebestimmung mit Carbazol nach Dische (6). Diese Reaktion wurde ohne wesentliche Modifikationen nach der Originalmethode durchgeführt.

Hexuronsäurebestimmung mit Naphthoresorcin, modifiziert nach Pelzer und Staib (7): $250 \mathrm{mg}$ Naphthoresorcin wurden in $100 \mathrm{ml}$ dest. Wasser gelöst und 3 Min. lang unter kräftigem Schütteln auf $100^{\circ}$ erhitzt. Dann erfolgte Abkühlen im Wasserbad bei Zimmertemperatur, Filtrieren, Stehenlassen über Nacht bei $0-4^{\circ}$ im Kühlschrank. $2 \mathrm{~m} /$ Analysensubstanz, $2 \mathrm{~m} /$ Naphtoresorcin-Reagenz und $4 \mathrm{~m} / 40$-proz. $\mathrm{H}_{2} \mathrm{SO}_{4}$ wurden gut gemischt und $30 \mathrm{Min}$. lang auf $100^{\circ}$ erhitzt. Nach Abkühlen im Wasserbad wurden $1 \mathrm{ml}$ absol. Äthanol und $4 \mathrm{~m} l$ Toluol zugegeben. Genau 15 Sek. lang exfolgte kräftiges Extrahieren. Nach Zentrifugieren wurde die organische Phase in $1 \mathrm{~cm}$-Küvetten bei Filter S $57 \mathrm{E}$ im Elko-Photometer (Fa. Zeiss) gegen Toluol gemessen. Eichkurve mit Glukuronolacton.

Bestimmung der Sauren Mucopolysaccharide im Harn Es wurde die Methode von Di Ferrante und Rrch (8) in der Modifikation nach TELLER (3) verwandt: nach Fällung der SMP mit Cetavlon bei pH 5,0 wurde das Präzipitat mehrmals mit 95-proz. NaCl-gesättigten Äthanol gewaschen und in $0,05 \mathrm{~N} \mathrm{NaOH}$ aufgenommen. In aliquoten Teilen wurde die Hexuronsäurebestimmung mit Carbazol $(1 \mathrm{ml})$ und Naphthoresorcin $(2 \mathrm{ml})$ durchgeführt.

\section{Ergebnisse}

$\mathrm{Da}$ die Hexuronsäurebestimmung mit Naphthoresorcin gewisse Störanfälligkeiten zeigte, prüften wir zunächst verschiedene Reaktionsbedingungen.

\section{Abbängigkeit der Farbentwicklung von der Dauler der Er- bitzung}

$40 \mu \mathrm{g}$ Chon rroitinsulfat B wurden nach Zugabe von Naphthoreso'cin-Reagenz im Doppelansatz 30, 60, 90 und $120 \mathrm{Mir}$. lang auf $100^{\circ}$ erhitzt. Die anschließende Extraktion t.nd Messung exfolgte wie bei der Originalmethode. Die Extinktionen, einschließlich der Reagenzienleerwerte, wurden gegen Wasser gemessen. Die Ergebnisse sind in Abbildung 1a dargestellt. Obwohl mit längerer Erhitzung eine zunehmende Menge CSB hydrolysiert und als Hexuronsäure meßbar wurde, fand die relativ stärkste Farbentwicklung nach 30min. Erwärmung statt. Diese Zeit schien somit für eine Chondroitinsulfat B-Bestimmung ausreichend zu sein.

\section{Abbängigkeit der Farbentvicklung von der Schwefelsäure- Konzentration ,."}

Zur Hydrolyse vor $40 \mu \mathrm{g}$ Chondroitinsulfat B wurde imi Doppelansatz Scínefelsäure in 15, 30, 40 und 50-proz. Konzentration vervandt. Als Vergleich dienten $10 \mu \mathrm{g}$ Glukuronolacton. Alle Proben, einschließlich der Reagenzienleerwerte, virden gegen Wasser gemessen. Die Ergebnisse sind in ibbildung $1 \mathrm{~b}$ graphisch dargestellt. Bis zu $40 \%$ konnte mit steigender $\mathrm{H}_{2} \mathrm{SO}_{4}$-Konzentration eine verstärkte Farbentwicklung erzielt werden; dann blieben die Extinktionen sowohl für Glukuronolacton als auch für CSB konstant. Daher wurde zur routine mäßigen Chondroitinsulfat B-Bestimmung eine 40-proz. $\mathrm{H}_{2} \mathrm{SO}_{4}$ gewählt.

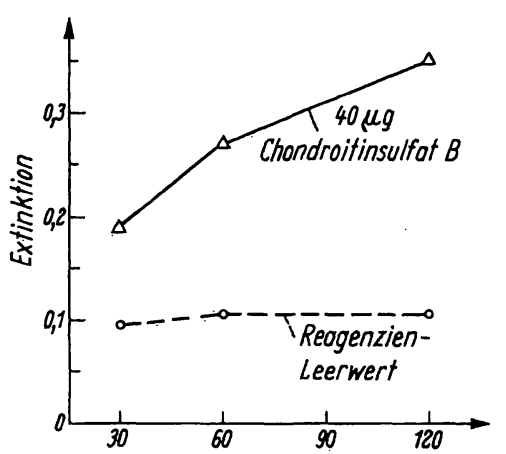

a Daucr der Erwärmung $\left(100^{\circ}\right)$ in $\mathrm{Mi}$.

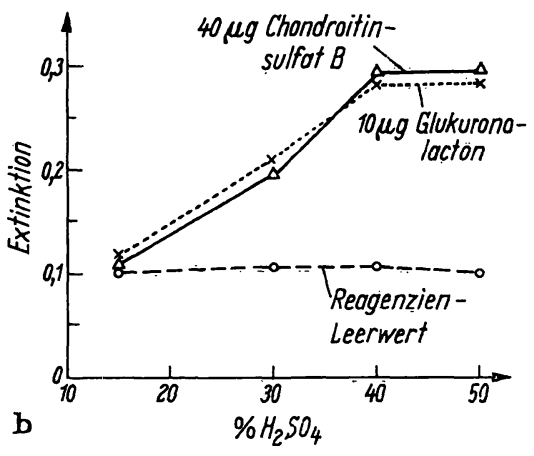

Abb. 1

Verschiedene Reaktionsbedingungen zur Hexuronsäurebestimmung mit der Naphthoresorcin-Methode

a) Abhängigkeit von der Dauer der Erhitzung; b) Abhängigkeit von der $\mathrm{H}_{2} \mathrm{SO}_{4}$-Konzentration.

Um Anhaltspunkte über die Empfindlichkeit der Carbazol- und der Naphthoresorcin-Methode zur Hexuronsäure- bzw. Mucopolysaccharid-Bestimmung zu gewinnen, wurden in beiden Reaktionen die spezifischen Extinktionskoeffizienten von drei Hexuronsäuren (DGlukuronsäure, L-Iduronsäure, D-Galakturonsäure) und vier Mucopolysacchariden (Chondroitinsulfat A, B und C, Heparitinsulfat) ermittelt. Die Ergebnisse sind in der Tabelle 1 zusammengestellt. Bemerkenswert war die ge-

Tab. 1

Spezifische Extinktions-Koeffizienten von Hexuronsäuren und sauren Mucopolysacchariden

$\left[\frac{\mathrm{E} \times(\mathrm{g} / \mathrm{m})^{-1}}{(\mathrm{~cm})}\right]\left[\frac{\mathrm{E} \times(\mathrm{g} / \mathrm{m})^{-1}}{(\mathrm{~cm})}\right] \begin{gathered}\mathrm{C} / \mathrm{N} \\ \text { Quotient }\end{gathered}$

\section{Hexuronsäuren}

D-Glukuronsäure 'L-Iduronsäure
D-Galakturonsäure

16200

4050

13300

Saure Mucopolysaccliaride

Chondroitinsulfat A

Chondroitinsulfat B

Heparitinsulfat
2300
970
2300
2600

2300

2600
Chondroitinsulfat $C$
12000

21500

22500

1,35

$0 ; 19$

0,59

10

0,6

12

$\begin{array}{cc}i 620 & 0,6 \\ 190 & 12 \\ 240 & 11\end{array}$


ringe Farbentwicklung von L-Iduronsäure und Chondroitinsulfat B in der Carbazol-Reaktion, obwohl diese Substanzen in der Naphthoresorcin-Reaktion ausgeprägte Chromogenitäten zeigten. Die Quotienten der spezifischen Extinktionskoeffizienten in den beiden Farbreaktionen (Carbazol/Naphthoresorcin- oder C/NQuotienten) verhielten sich bei den Hexuronsäuren wie folgt: D-Glukuronsäure > D-Galakturonsäure > LIduronsäure. Diejenigen Mucopolysaccharide, die als Hexuronsäure $\mathrm{D}$-Glukuronsäure enthalten, zeigten in beiden Reaktionen annähernd gleiche spezifische Extinktionskoeffizienten, während Chondroitinsulfat B, das aus L-Iduronsäure besteht, eine stärkere Farbentwicklung in der Naphthoresorcin-Reaktion aufwies. Der $\mathrm{C} / \mathrm{N}$-Quotient war nahezu $20 \mathrm{fach}$ niedriger als bei den drei anderen Mucopolysacchariden.

In einem Mucopolysaccharid-Gemisch aus Heparitinsulfat und Chondroitinsulfat $A$ und $C$ konnte bei konstanter Gesamtmenge die Farbentwicklung in der Carbazol-Reaktion durch steigenden Zusatz von Chondroitinsulfat $B$ linear vermindert werden, während in der Naphthoresorcin-Reaktion der CSB-Zusatz zu einem linearen Farbanstieg führte (Abb. 2). Für jeden beliebigen Anteil von Chondroitinsulfat B an der Mucopolysaccharid-Gesamtmenge ließen sich $\mathrm{C} / \mathrm{N}$-Quotienten ermitteln, die zueinander in einfacher exponentieller $\mathrm{Be}-$ ziehung standen und im halblogarithmischen Koordinatensystem eine Gerade bildeten (Abb. 3).

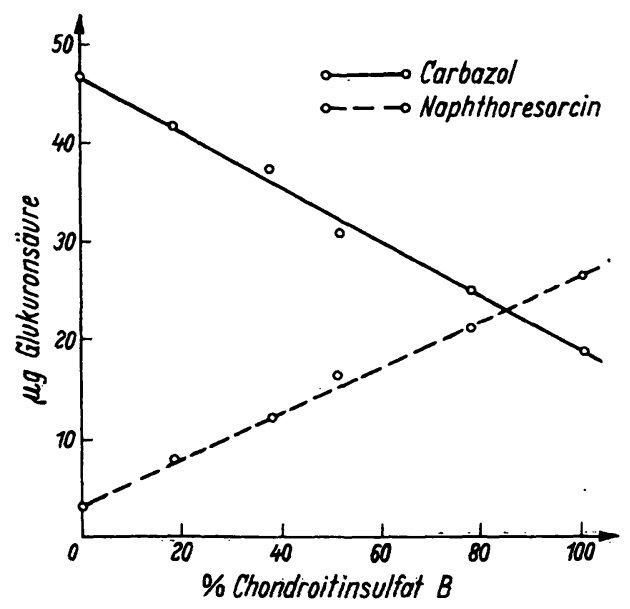

Abb. 2

Der Einfluß steigenden Chondröitinsulfat B-Gehaltes auf die Farbentwicklung eines Gemisches sauter Mucopolysaccharide in der Carbazol- und der Napbthoresorcin-Reaktion

Bei der Bestimmung der C/N-Quotienten der GesamtMucopolysaccharide im Harn von 37 Normalpersonen verschiedenen Alters ergab sich ein Mittelwert von 15,1 $\pm 5,6(M \pm \sigma) .13$ Patienten mit typischer Pfaundler-Hurler'scher Krankheit wiesen einen durchschnittlichen $\mathrm{C} / \mathrm{N}$-Quotienten von 4,2 $\pm 1,2(\mathrm{M} \pm \sigma)$ auf. Dieser Wert lag statistisch signifikant unterhalb der Norm und ist auf den erhöhten Anteil von Chondroitinsulfat B am Gesamt-Mucopolysaccharid-Material im Harn von Patienten mit Gargoylismus zurückzuführen.

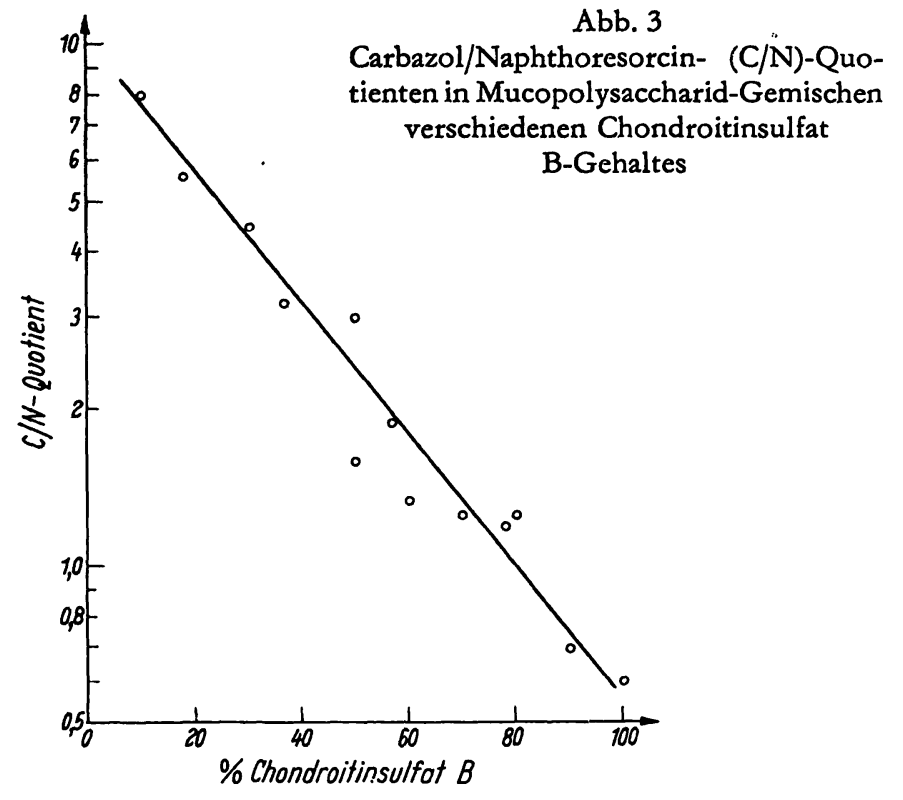

\section{Diskussion}

Verschiedene Autoren haben bei der Bestimmung der Neutralzucker und Hexuronsäuren in Mucopolysacchariden versucht, die Reaktionsbedingungen so zu variieren, dạ $\beta$ für jedes Mucopolysaccharid typische Farbentwicklungen auftreten. Dabei verwandten HeLBERT und Brown (9) die Anthron-Methode. RadhakrishNAMURTHY und BERÉnson (5) ermittelten in der Carbazol-Reaktion durch Veränderung der Erwärmungstemperatur und -dauer die Bedingungen, bei denen L-Iduronsäure oder Chondroitinsulfat B eindeutig andere Extinktionen als D-Glukuronsäure bzw. Chondroitinsulfat A ergeben. Die Anwendung dieses Verfahrens auf die qualitative Untersuchung der Mucopolysaccharide im Harn schien nach Ansicht der Autoren eine Aussage über den relativen Chondroitinsulfat BGehalt zuzulassen.

Im Anschluß an die in unserem Laboratorium routinemäßig durchgeführte Bestimmung der sauren Mucopolysaccharide im Harn (3) können diejenigen Proben, die einen eindeutig erhöhten Carbazol/Kreatinin-Quotienten aufweisen, durch die anschließende NaphthoresorcinReaktion ohne größeren Aufwand auch qualitativ hinsichtlich Chondroitinsulfat $B$ analysiert werden. Ergibt

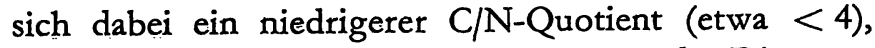
so bestehen nach unseren Erfahrungen an der Diagnose eines Gargoylismus keine ernsthaften Zweifel mehr. Schwieriger wird die biochemische Diagnostik der verschiedenen Untergruppen des Gargoylismus. Sie gehen nämlich teilweise nicht mit einer prozentual erhöhten Chondroitinsulfat B-Ausscheidung einher, sondern weisen größere Mengen Heparitinsulfat im Harn auf. $r$.ie $\mathrm{C} / \mathrm{N}$-Quotienten sind in diesen Fällen normal. Auch die aus Keratosulfat bestehende Mucopolysaccharidurie der Morquioschen Krankheit kann mit der beschriebenen Methode nicht erfaßt werden. Hier wird nur durch die chromatographische Fraktionierung der Mucopolysaccharide eine weitere Klärung möglich sein $(4,10)$. 


\title{
Literatur
}

1. Dorfman, A. und A. E. Lorincz, Proc. nat. Acad. Sci. (Wash.) 43, 443 (1957). - 2. MEYER, K., M. M. GRUMBACH, A. LiNKER und P. Hoffman, Proc. Soc. exp. Biol. Med. 97, 273 (1958). - 3. Teller, W., Mschr. Kinderhk. 113, 244 (1965). - 4. Maroteaux, P. und M. LaMr, J. Pediatr., S. Louis 67, 312 (1965). - 5. Radhakrishnamurthy, B. und G. S. Berenson, Analytic. Chem. 35,
1316 (1963). - 6. Drsche, Z., J. biol. Chemistry 167, 189 (1947). - 7. Pelzer, H. und W. Staib, Clin. chim. Acta (Amsterdam) 2, 407 (1957). - 8. Di Ferrante, N. und C. Rich, J. Laborat. clin. Med., S. Louis 48, 491 (1956): - 9. HeLbert, J. R. und K. D. Brown, Analytic: Chem. 33, 1610 (1961). - 10. Teller, W. und A. ZIEMANN, Klin. Wschr. 44, 1142 (1966).

Privat-Dozent Dr. med. W. Teller Universitätś-Kinderklinik 355 Marburg/Lahn

\section{Darstellung der Haptoglobintypen durch Elektrophorese in Polyacrylamidgel}

Von F. Schleyer und P. Schaible

\begin{abstract}
Aus dem Institut für gerichtlicbe Medizin der Universität Marburg (Direktor: Prof. Dr. F. Schleyer)
\end{abstract}
(Eingegangen am 21. Juni 1966)

\begin{abstract}
Es wird eine Abwandlung der Acrylamidgel-Elektrophorese als geschlossenes System zur optimalen Darstellung der Haptoglobintypen im menschlichen Serum beschrieben. Für die Aufnahme der Gelsäulen wurde eine besondere Färbewanne konstruiert. Die entstehenden Bandenspektren erlauben eine hervorragende Differenzierung der Phänotypen. Bei Typ 2-1 konnten bis zu 13 Banden gezählt werden.

A modification of acrylamide gel electrophoresis is described, which employs a closed system and gives an optimum separation of haptoglobin types in human sera. A special dish was constructed for staining the gel columns. The band spectra produced by this method permit an excellent differentation of phenotypes. Up to 13 bands could be detected in type $\mathrm{Hp} 2-1$.
\end{abstract}

BAUMgarten (1) hat im Jahre 1963 als erster Autor das Acrylamid als Gelmedium zur speziellen Darstellung der Hp-Typen empfohlen. Gervars und Vescou (2) haben die Methode zur Hp-Diagnostik an Blutfleckenauszügen erprobt. Im folgenden wird eine einfache Standardtechnik beschrieben, die eine hervorragende Hp-Typendarstellung erlaubt. Die physikalisch-chemischen Grundlagen und Methoden der Acrylamidgel-Elektrophorese findet man $u$. a. bei $(3-10)$ beschrieben.

\section{Methodik}

Herstellung des Gels: Als optimale Polymerkonzentration ermittelten wir einen Acrylamidgehalt von 5,5\% und eine Vernetzerkonzentration ( $\mathrm{N}, \mathrm{N}^{\prime}$-methylenbisacrylamid, ,MBA ${ }^{c}$ ) von $5 \%$, bezogen auf Acrylamid (,AA“). Dieses Verhältnis ergibt offenbar den für die $\mathrm{Hp}-$ Molekülgröße günstigsten Porendurchmesser und trennt die Hp-Banden bei der gewählten Trennzeit am weitesten und schärfsten. Die durch den relativ hohen MBA-Gehalt bedingte Opaleszenz stört nicht. In dem Präparat „Cyanogum 41“ der "Cyanamid Company" sind die Monomeren gebrauchsfertig gemischt; der MBA-Gehalt dürfte, der Opaleszenz nach zu urteilen, bei $4-5 \%$ liegen. Die Ergebnisse sind gleich. Als Katalysatoren wählten wir $\beta$-Dimethylaminoproprionitril („DMAPN") und Ammoniumpersulfat. Das optimale Mischungsverhältnis der Komponenten ist (in dieser Reihenfolge):

$\begin{array}{ll}\text { Gelpuffer (s. u.) } & 94,0 \\ \text { AA } & 5,5 \\ \text { MBA } & 0,275 \\ \text { DMAPN } & 0,1 \\ \left(\mathrm{NH}_{4}\right)_{2} \mathrm{~S}_{2} \mathrm{O}_{8} & 0,1\end{array}$

Jede Komponente muß vor Zugabe der nächsten vollständig gelöst sein. Nach 25-30 Min. (bei Konzentrationen der Katalysatoren von je $0,4 \%$ schon nach $2-3$ Min.) ist das Gel gebrauchsfertig.
Einfüllen des Gels: Wir arbeiteten in einem gescblossenen, vertikalen Zylindersystem. In senkrecht gestellte Präzisionsglastöhren $(13,0 \times 0,5 \mathrm{~cm})$, die eine Nummer und bei $10,5 \mathrm{~cm}$ eine Marke tragen und am unteren Ende zunächst mit einem Serumstopfen verschlossen werden, pipettiert man etwa $2,3 \mathrm{~m} l$ des noch flüssigen Gemisches bis zur Marke. Um bei der Polymerisationszeit von $30 \mathrm{Min}$., die sich bei Anfall einer größeren Serumprobenzahl empfiehlt, eine glatte Abschlußfläche des Gels zu erzielen, gibt man oben 2 Tropfen Wasser hinzu. Die vollendete Erstarrung ist an einer leichten Opaleszenz zu bemerken. Das überstehende Wasser wird abgegossen.

Beschicken des Gels: Nunmehr werden in jede Röhre $0,02 \mathrm{ml}$ des in der üblichen Weise bereiteten Serum-Hp-Gemisches eingetropft. Sodann werden $2-3$ Tropfen einer schnellpolymerisierenden, d. h. mit je 0,4\% der Katalysatoren-Komponenten zubereiteten Monomerenmischung (oder eines solchen Cyanogum-Ansatzes) zugegeben. Ist dieses Verschlußgel fest, so wird die überstehende Flüssigkeit abgegoșsen.

Das Elektrophoresegerät: Wir konstruierten ein $3 \mathrm{~cm}$ tiefes, zylindrisches, offenes Kathodengefä $\beta$ von $12 \mathrm{~cm} \varnothing$ aus Trovidur (Hersteller: Fa. Kniese, 355 Marbach bei Marburg). Der Boden dieses Aufsatzes enthält in kreisförmiger Anordnung zwei Reihen von insgesamt 16 leicht konischen Bohrungen von $0,12 \mathrm{~cm} \varnothing$ für die Aufnahme der Röhrchen. In den Bohrungen stecken passend durchbohrte Gummistopfen. Durch die Mitte des Gefäßbodens fühtt in einem Trovidur-Rohr die Zuleitung zur Anode. Dem Aufsatz ist ein $13 \mathrm{~cm}$ hohes und $10 \mathrm{~cm}$ weites Standgefäß aus $G$ las eingepaßt. Auf seinem Boden kommt die Anode zu liegen (Abb. 1). -Nachdem die Stopfen am unteren Röhrenende (ohne Sog!) entfernt wurden, werden die gefüllten Glasröhren soweit durch die durchbohrten Gummistopfen im Boden des Kathodenaufsatzes eingedreht, daß sie gerade mit dem oberen Stopfenende abschließen. Sodann wird so viel Elektrodenpuffer (s. u.) in den Glaszylinder eingefüllt, daß die Röhren etwa $0,5 \mathrm{~cm}$ tief eintauchen. Die Anode wird durch einen Steckkontakt mit der Zuleitung im TrovidurRohr verbunden, und der Aufsatz mit den Röhren eingesetzt. Auf die Gelsäulen in den Röhrchen wird Puffer bis zum Uberlaufen 